\title{
Conferencia Inaugural II Congreso Chileno de Salud Pública Desafíos para la salud pública contemporánea en tiempos de cambio
}

PAULO BUSS. PRESIDENTE DE LA FEDERACIÓN MUNDIAL DE ASOCIACIONES DE SALUD PÚBLICA

Tuve muchas dificultades para enfrentar este tema de "Desafíos para la salud pública contemporánea en tiempos de cambio", en el marco del Bicentenario. Lo abordaré desde el punto de vista de la salud, desarrollo y sociedad, y cuál es el rol de la salud pública en este contexto. Trabajaré los conceptos de prácticas de la salud pública, cuáles son sus funciones esenciales hasta hoy y, finalmente, hablaré sobre los cambios políticos y sociales que estamos viviendo en el umbral del siglo XXI en todo el mundo, y que influyen directamente en la salud pública, además de discutir el tema de la salud pública global y una subsalud que, a mi juicio, es la salud pública a nivel subregional de América del Sur. Finalmente, me referiré a los desafíos contemporáneos de la salud pública.

Los conceptos y prácticas de salud, salud pública y sistemas de salud han cambiado a lo largo del tiempo en cualquiera que sea la formación social que se considere, y existen tantas nociones de salud pública en cuanto a coyunturas y formaciones sociales se refiere. No pretendo entregar una receta de nada, porque la salud pública tiene esa característica de estar involucrada en su tiempo; es una disciplina en la práctica histórica, pues tiene que ver con el territorio no como geografía sino como proceso social, y con la episteme que uno vive.

Entonces, los riesgos que afectan a la salud, las enfermedades, así como los cuidados individuales y colectivos, es decir, a la gente enferma o a la gente sana, y colectivos en salud, son socialmente producidos. Salud es un producto biológico pero también, creo, un producto social. Todas las evidencias construyen que los conceptos y prácticas de salud públi- ca son indisociables de la organización social, sea política, poder, ideología y cultura, y del conocimiento de diversos temas. Es decir, es un producto biológico-social, y en el sentido social que depende de la organización social y del conocimiento.

Son categorías técnico-científicas, una categoría histórica y una categoría política. Aquí cabe nombrar al maestro Vicente Navarro, quien dice: tanto el conocimiento científico de lo que es salud/enfermedad como lo que constituye lo que se define como práctica médica y salud pública, y las que se reconocen como instituciones sanitarias, están altamente influenciadas por el contexto social y político. Y Jairnilson Paim escribió: el carácter histórico y estructural de la salud pública como campo de saber y práctica permite que cada formación social engendre su propia salud pública. Ese es un desafío para los chilenos y para cada uno de nosotros.

Salud pública es el campo de conocimientos y prácticas, cuyos compromisos sociales e históricos son la identificación de las condiciones de salud de la población y de sus determinantes, así como la formulación e implementación de políticas que conllevan intervenciones resolutivas y para mejorar, promover, proteger y restaurar la salud de la población, acompañándose de la movilización de diversas fuerzas sociales y sectores gubernamentales. Es decir, la salud pública es un trabajo que está en manos del Estado prioritariamente. Además, conlleva el concepto de que salud es parte de un sistema de protección social, que es un sistema público, y que debe partir por la defensa de la salud y de la calidad de vida de la población.

Los enfoques prioritarios de la salud públi- 
ca idealmente en una reformación social inserta en el espacio/territorio - el territorio en proceso y en determinada coyuntura- dependen de determinada concepción del proceso salud/ enfermedad vigente, y sus determinantes. Y también de la identificación de recursos organizacionales, tecnológicos y financieros estatales, públicos y comunitarios, más eficaces y eficientes, para enfrentar de forma satisfactoria las presiones del proceso y sus determinantes. Esa es una conceptualización importante que me gustaría compartir con ustedes.

Vemos otra dimensión aquí entonces. La salud pública identifica los procesos determinantes de la situación de salud y organiza formas más resolutivas de actuar sobre esos procesos determinantes, de forma de mejorar la salud y la calidad de vida de los individuos y de la población como un todo. Y haciendo un llamamiento a nuestra experiencia cotidiana de salubristas, se utilizan instrumentos de la epidemiología y de la planificación, administración y organización del sistema y de los servicios de salud, así como de la vigilancia en salud, sea la vigilancia epidemiológica, sanitaria o ambiental, utilizando acciones articuladas de promoción de la salud, prevención de enfermedades y de asistencia a los enfermos. Y para ser efectiva tiene necesariamente que incluir la articulación con otros sectores gubernamentales y con la sociedad.

Entonces, la salud pública es parte integrante del sistema de salud a pesar de que todos los procesos de reforma impartidos desde el Banco Mundial, desde los organismos del capitalismo central, nos dicen que los procesos de reforma deben tratar de resolver el problema de la atención médica. En todos los procesos de reforma que se proponen, la salud pública está fuera. Está olvidada, destacando que los sistemas de salud son un conjunto de intervenciones en la sociedad cuyo principal propósito debería ser la salud.

Hoy, hay un tremendo consenso de que la importancia de las acciones sobre los determinantes de la salud y determinantes sociales de la salud se encuentra bajo el liderazgo intransferible del Estado. El Estado tiene que tomar cuenta de la salud pública, es responsable de la salud colectiva y eso me parece que es algo en lo que tenemos que hacer hincapié desde siempre. Por lo tanto, el sistema de salud es más amplio que el sistema de atención o de los servicios de atención a los cuales incluye.

Finalmente, debo tomar la definición de la Organización Panamericana de la Salud (OPS), que dice que las políticas y acciones de salud pública deben necesariamente tomar en cuenta los contextos que las involucran y las determinan, esto es, los contextos político, económico, social y ambiental, así como las condiciones de salud y sus tendencias, como por ejemplo, las características de la población, la magnitud de distribución de tendencias de la mortalidad, la morbilidad, incluyendo las enfermedades no transmisibles, las causas externas, etc.

¿Y los actores de la salud pública? Insisto en el Estado como el principal actor institucional de la salud pública y que organiza su actuación a través de instituciones estatales como ministerios, secretarías nacionales, provinciales y municipales de salud; es decir, las autoridades sanitarias que lideran proyectos de naturaleza pública en cada esfera de gobierno o en cada nivel de gobierno. Es muy importante este tema porque en la mayoría de los sistemas de salud tenemos procesos de descentralización política; entonces, es importante la dirección compartida entre sectores. Por sus sujetos y campo de práctica, la salud pública es parte funcional y operativa integrante del sistema de salud, y tiene identidades profesionales y operativas específicas. Y ahí podríamos incluir un concepto que se discute en los últimos 10 años, de las funciones de la salud pública, que serían: el análisis, monitoreo y evaluación de la situación social y de salud. Y lo pongo sólo para que recordemos esa contribución conceptual para la salud pública. Segunda función: la vigilancia, investigación y control de riesgos de daños en salud no solo correspondiente a las enfermedades infecciosas como era la tradición de principios del siglo XX, pues ahora tenemos un montón de nuevos riesgos de daños en salud, inclusive los sociales. La promoción de la salud, la participación social en sa- 
lud, la formulación de políticas, planificación y gestión de sistemas de servicio. La vigilancia sanitaria, es decir, la regulación y fiscalización sobre bienes, servicios y productos, que es una función noble de la salud pública, porque no puede hacerla cada agente individualmente: la calidad del agua, de los productos, del ambiente en general; esa es una función de la salud pública muy importante, porque creo que ahí se ubica un nuevo desafío para nosotros, que es la promoción de la equidad del acceso a los servicios.

La equidad es el tema central que domina el debate internacional y nacional en la mayoría de los países del mundo, el desarrollo de los recursos humanos, la garantía de calidad de los servicios, la investigación en salud pública, y el tema de las emergencias y desastres. En ese sentido, Chile tiene una experiencia como país, compleja, después de los últimos acontecimientos ocurridos. En la publicación de la OPS, "La salud pública en las Américas, nuevos conceptos", de 2002, se encuentra una amplia discusión sobre estos temas.

Me gustaría compartir con ustedes algunas visiones sobre esas nuevas realidades en el umbral del siglo XXI, y que nos conducen a preguntar cuál es el rol de nosotros, los salubristas y la salud pública, en éstas. Tenemos frente a la discusión una crisis económica, sistémica y global importante del capitalismo, del modelo capitalista. Creo que no es solamente una crisis coyuntural, es una crisis básica de modelo, que empezó en 2008 y del circuito central de una economía globalizada, que dos años después continúa produciendo consecuencias impresionantes sobre la economía global, por la calidad de vida, sobre su población y sobre la salud. Esta crisis ha profundizado las desigualdades persistentes y ha causado una amplificación de la pobreza y del desempleo. Además, hoy existe un compromiso ecológico en escala planetaria, el deterioro medioambiental, cuyas consecuencias sobre la calidad de vida, la producción agrícola, la vida en sí, son impresionantes. Esto nos está amenazando no sólo a nosotros sino también a nuestros hijos, nietos y a las futuras generaciones. Ese sería el componente ético transgeneracional que tiene nuestra generación. Nosotros no podemos imaginar las consecuencias futuras de lo que estamos haciendo hoy, y más que eso, de lo que No estamos haciendo hoy para contener el deterioro medioambiental. Entonces, ese es un imperativo ético.

Este tema del compromiso ecológico me parece central y está muy lejos, en general, de la agenda de la salud pública; incluso, nos parece que está tan lejos que pensamos que no tenemos nada que hacer. Como resultado del calentamiento global, tenemos una importante crisis alimentaria, que también se debe a que dos países empezaron a consumir más alimentos: China e India. Debemos reflexionar al respecto. También sufrimos una crisis energética, vinculada a decisiones que tenemos que tomar hoy sobre el futuro del patrón energético de nuestros países, con los resultados que esto tiene sobre el medio ambiente. Y además, vivimos una crisis ética, porque no es ético que un pequeño grupo de inversores de Wall Street produzcan una crisis de las dimensiones que hoy estamos viviendo; eso es éticamente inaceptable.

Todo ello con consecuencias que son muy graves sobre los países de ingresos bajos y sobre los estados frágiles; es decir, más de 70 países, con sociedades y estados frágiles, que son casi todas las naciones africanas y muchas decenas de países de Asia. Ellos no tienen capacidad de reacción y es ahí donde la crisis impacta más fuertemente.

Así, vivimos tiempos de mucha incertidumbre, pero también hay cambios políticos importantes y positivos. Por ejemplo, la creciente conciencia y reconocimiento social de los pueblos originarios y de las diversidades culturales, éticas, de género, de modo de vida, además del impacto de otras determinantes sociales de la salud. El tema hoy es la diversidad y la interculturalidad. Las lecciones que estamos obteniendo de los países andinos son impresionantes, en cuanto al bien vivir de los indígenas. Tenemos que pensar profundamente acerca del tema de la promoción de la salud bajo conceptos innovadores antiguos como el 
bien vivir, como piensan los indígenas de la región de las Américas. Creo que ese es el punto positivo en este tiempo y que impacta en la salud pública.

También es muy importante la reinvención del Estado en el desarrollo. Me impresiona que los países que en los años 90 y en los primeros cinco años de los 2000, antes de la crisis, decían: el Estado tiene que ser disminuido -como la Unión Europea y Estados Unidos, el Estado más poderoso de la Tierra- hacen una intervención impresionante con el dinero público, con nuestro dinero, con el dinero de ellos, pero que impacta sobre nosotros. Cuando Estados Unidos decide defender la moneda desvalorizando el dólar, eso tiene impacto sobre nuestras exportaciones y, por lo tanto, sobre las riquezas que podemos obtener; entonces, eso se tiene que pensar, porque es parte del impacto en la calidad de vida y por lo tanto en la salud de las personas.

En la reinvención del Estado en el desarro1lo, nosotros en Sudamérica debemos dar un ejemplo. Cuando tenemos la confianza y el valor de la autodeterminación nacional, una democracia renovada, tenemos ejemplos de los países andinos, de Brasil, Chile y Uruguay, donde hace poco hubo elecciones libres, una reafirmación de la democracia y lo mismo sucedió en muchas democracias africanas donde había dictadores terribles. Esta reactivación del proceso democrático me parece sumamente importante porque nos garantiza, sobre todo a partir de la política, calidad de vida y también los impactos políticos que tenemos sobre la salud.

Ahora, ¿qué podemos decir sobre los perfiles que vivimos hoy con cambios, tensiones, diferencias entre nuestros países? Trabajaremos primero con esas que son las determinantes sociales de la salud, donde podemos poner algunas ideas de cómo se encuentra la pobreza y la exclusión social en nuestros países. Y los estudios son muy importantes, porque las medias esconden grandes inequidades, incluso en países con indicadores de salud mejor, como los que tiene Chile. Si vamos desglosando en el territorio, por clases sociales, vemos cuánta inequidad tenemos aquí como en Brasil y en otros países. Entonces, los temas de la pobreza y la exclusión así como el de la creciente urbanización, con todo el peso que eso tiene sobre el medio ambiente, la industrialización, influyen en el medio donde vive la gente. En este punto, me impresiona que hasta el momento nosotros no logremos dar atención al tema de la migración y de los inmigrantes, porque una cosa es el fenómeno de la inmigración y otra cosa son los inmigrantes, los seres humanos involucrados. En Sao Paulo hay muchos inmigrantes en las peores condiciones de salud pública que se puedan imaginar. El envejecimiento de la población tiene que ver con la fecundidad y con el crecimiento poblacional. Chile es un ejemplo que empezó más temprano que en otros países, pero hoy Brasil tiene un $1,9 \%$ de fecundidad y no crecemos más. Pero es importante, insisto, preocuparse de las inequidades sanitarias entre los países y dentro de los países, y la situación declinante de salud en muchos países del mundo.

Tenemos un desafío importante con los virus del dengue, el virus H1N1, las enfermedades crónicas no trasmisibles, las enfermedades mentales que, a mi juicio, representan otro punto bastante olvidado en el trabajo en salud pública; las dificultades de inserción de la gente en sociedades que cambian rápidamente; y las violencias -no solo interpersonales sino también las violencias institucionales-, como las de las guerras y las intervenciones de un Estado en otro Estado, con su consecuencia de miles de muertes. Ahora estamos trabajando una resolución sobre guerra en la Asociación Internacional sobre Salud Pública, porque aquí hay miles de muertes de jóvenes y de civiles en países como Afganistán e Irak, y eso tiene que ver con salud pública. Finalmente, hay una discusión sobre otros temas que nos deben preocupar, como son las adicciones y los estilos de vida no saludables con la paradoja desnutrición/obesidad que conviven; y si desglosamos ambos temas, vamos a verificar dónde se ubican esas situaciones. Y eso es una inequidad terrible para mí, porque tanto los obsesos como los desnutridos, están en la población 
más pobre, no en la más rica, que tiene posibilidad de acceso a un alimento de mejor calidad y también tiene más acceso a la información.

Por otro lado, está la globalización de los riesgos en salud y también lo que pasó con Chile debido al terremoto, que tiene que ver con la vulnerabilidad frente a los desastres y a otras circunstancias de impacto ambiental. La salud pública tiene que estar preparada para eso y, a veces, no lo está.

Y tenemos graves problemas en los países de renta media y baja, donde hay limitaciones en los gobiernos, en sistemas de salud frágiles, en la fuerza de trabajo y que sufren la migración de los profesionales hacia los países que pueden pagar más; que tienen inadecuados recursos tecnológicos, que están subfinanciados y que son muy frágiles el control social o simplemente no pueden cumplir con él. Esos países sufren esos perfiles epidemiológicos de los cuales hablé antes y tienen este problema en sus sistemas de salud. También está el problema de los insumos en salud, por ejemplo, tenemos hoy un imperio de las patentes sobre los pacientes, en el cual los medicamentos, las vacunas, los recursos para diagnóstico, incluso los equipos, son muy caros para la mayoría de los países y su precio no es por el contenido del medicamento, sino por el tema de las patentes. Y en la patente está involucrado el lucro que las empresas tienen; por eso, nosotros estamos en constante lucha contra el tema de las patentes por sus consecuencias en los pacientes.

Las consecuencias están ahí, pero aquí toma especial relevancia la cooperación internacional y por eso trabajaré un poco ahora con el concepto de Salud Pública Global y Diplomacia de la Salud. Me parecen dos temas importantes.

Ya hemos constatado la importancia de la dimensión internacional de la salud, pero debemos decir que hay una crisis importante en la gobernabilidad. Por ejemplo, hay tensiones importantes entre la Organización Mundial de la Salud, la Organización Mundial del Comercio y la Organización Mundial de la Propiedad Intelectual, porque ahí está el corazón del capital, donde están los intereses de las empresas privadas.

Me permito recordar el surgimiento de nuevos actores globales. Son las tantas organizaciones que están trabajando solo con el tema de las molestias defectuosas parasitarias, consumiendo una gran parte de los recursos en sus burocracias y la plata no llega muchas veces a la gente que más lo necesita. Por ejemplo, pasa con la malaria, las vacunas, etc. Por otro lado, la proliferación y la descoordinación de la cooperación de los donantes; la cooperación vertical a proyectos, a programas, y no a reforzar los sistemas de salud, son problemas serios de la cooperación internacional de salud. Y una reducción muy importante de la ayuda externa. Solo cinco países ofrecen el $0,7 \%$ de su producto interno bruto al desarrollo, a la cooperación internacional para el desarrollo, que es la gran conquista de la Cúpula del Milenio con el compromiso del 0,7\%. Sólo Noruega, Luxemburgo, Dinamarca, Suecia y los Países Bajos lo hacen. Y cuando comparamos esto con los gastos militares, la diferencia es brutal.

Las Naciones Unidas hicieron varias conferencias durante el año 90 con el objetivo de preparar al mundo para el siglo XXI. Las menciono porque si vemos las conferencias que están en internet, vemos qué maravillosos son los análisis y las propuestas; planteo que si sólo el 10\% de lo que se propone en esas conferencias estuviera hecho, nosotros estaríamos en otro mundo, pero creo que es importante que nos inspiremos en esos documentos, porque fueron hechos con muy buena voluntad. Y ahí tuvimos la Cumbre, la Declaración del Milenio, los Objetivos del Desarrollo del Milenio, que yo los pongo aquí sólo para hacer una observación: que hay gente que desprecia los objetivos de salud para el milenio, pero éste ha sido el único acuerdo posible entre los 191 países. Y si miramos bien los objetivos, tenemos que esos son determinantes sociales de salud en estado puro.

El Globalized Forbicis Immunization es otro ejemplo que debo destacar para no quedarnos tan deprimidos, una forma muy interesante de cómo aportar vacunas básicas a más de 70 países, y lo hizo una alianza internacio- 
nal; también, la $4^{\mathrm{a}}$ Convención sobre control de tabaco es un impresionante acuerdo internacional al cual se llegó. El reglamento sanitario internacional lo mismo. Todos los países están impartiendo reglas comunes en términos del reglamento sanitario. Y me gustaría hacer dos menciones a documentos, que son muy importantes: uno, el documento de la comisión de la OMS sobre determinantes sociales de la salud y el informe de la OMS sobre atención primaria de salud, más necesaria que nunca, documentos que nosotros debiéramos trabajar y discutir, para pensar qué desafíos contemporáneos tiene la salud pública, qué camino se puede trazar para nuestros países y nuestra región, globalmente. Y pensar en la importancia de la cooperación sur-sur, que se da entre países en desarrollo, en forma horizontal, un proceso de asociación económica, comercial, social o de otra naturaleza, que se establece con ventaja mutua entre socios de países en desarrollo generalmente ubicados en el hemisferio sur. Aquí está subyacente al concepto la hipótesis de que el mundo en desarrollo es capaz de producir soluciones auténticas para sus propios problemas, como una cooperación entre semejantes.

Para cerrar, creo que salud pública a nivel regional, dentro de la cooperación sur-sur, y lo que llamamos la salud como una fuente para la paz, apuntando a la importancia de la salud, sería en el foco de pensar la unión de naciones sudamericanas. Es una reunión de dos grandes áreas: Mercosur y la comunidad andina. Y Chile pertenece a los dos conjuntos; entonces, su importancia ha sido importante. Unasur tiene un consejo de presidentes, un consejo de delgados, los consejos sectoriales, donde se incluye la defensa y la salud, entre otros. El Consejo de Ministros fue creado en diciembre de 2008 en Brasil, formado por los 12 ministros de salud de los países, y un comité coordinador donde están sus representantes. La reunión que instaló el consejo fue en 2009 y cinco equipos técnicos han desarrollado la agenda sudamericana de salud, y que tiene un representante por país. Chile y Brasil están presentes.

Finalmente, debo destacar que debemos reforzar las funciones esenciales de la salud pública, es decir, hacer la tarea en el hogar, en nuestra casa. Tenemos que construir una salud pública muy bien hecha por nuestros gobiernos, por nuestro personal de salud. Pero para eso debemos introducirla en el corazón de las reformas sectoriales, cobrando las transformaciones necesarias, demandando el dinero necesario para la formación de la gente, para que pueda dar su respuesta a nuestras sociedades. Es muy importante la acción intersectorial en las políticas públicas, para enfrentar los determinantes sociales de la salud pública.

Los sistemas de salud aislados no conseguirán resolver el problema de salud de nuestras comunidades. Por eso, son muy importantes las políticas públicas y una acción articulada intersectorial en esta materia, entre educación, salud, vivienda, transporte, agua; es decir, los más significativos componentes de la calidad de vida. Es muy importante que aprendamos cómo la gente vive y se conecta con su realidad para mejorar los programas y adaptarlos a las mayores necesidades que tienen nuestras sociedades.

La búsqueda constante de la equidad en salud, la decisión de superar las grandes desigualdades que encierran las medias y trabajar para que éstas disminuyan, tiene que ser un reto permanente y constante de la salud pública. Debemos promover los cambios en las formas de hacer las cosas, en el gobierno global, y buscar -tal como lo hizo hace un tiempo el gran salubrista chileno, Hugo Behm Rosasnuevos instrumentos y herramientas técnicas para hacer de la salud pública algo que las poblaciones respeten y apoyen. Muchas gracias. 